\title{
Nonlinear Evolution of Benjamin-Bona-Mahony Wave Packet due to an Instability of a Pair of Modulations
}

\author{
Vera Halfiani $\mathbb{D}^{1},{ }^{1}$ Dwi Fadhiliani, ${ }^{1,2}$ Harish Abdillah Mardi, ${ }^{1,3}$ and Marwan Ramli $\mathbb{D}^{1,4}$ \\ ${ }^{1}$ Dynamic Application and Optimization Research Group, Syiah Kuala University, Banda Aceh 23111, Indonesia \\ ${ }^{2}$ Mathematics Graduate Study Program, Syiah Kuala University, Banda Aceh 23111, Indonesia \\ ${ }^{3}$ Department of Physics, Syiah Kuala University, Banda Aceh 23111, Indonesia \\ ${ }^{4}$ Department of Mathematics, Syiah Kuala University, Banda Aceh 23111, Indonesia
}

Correspondence should be addressed to Marwan Ramli; marwan.math@unsyiah.ac.id

Received 1 January 2018; Accepted 19 April 2018; Published 3 June 2018

Academic Editor: Masaaki Tamagawa

Copyright (C) 2018 Vera Halfiani et al. This is an open access article distributed under the Creative Commons Attribution License, which permits unrestricted use, distribution, and reproduction in any medium, provided the original work is properly cited.

\begin{abstract}
This article discusses the evolution of Benjamin-Bona-Mahony (BBM) wave packet's envelope. The envelope equation is derived by applying the asymptotic series up to the third order and choosing appropriate fast-to-slow variable transformations which eliminate the resonance terms that occurred. It is obtained that the envelope evolves satisfying the Nonlinear Schrodinger (NLS) equation. The evolution of NLS envelope is investigated through its exact solution, Soliton on Finite Background, which undergoes modulational instability during its propagation. The resulting wave may experience phase singularity indicated by wave splitting and merging and causing amplification on its amplitude. Some parameter values take part in triggering this phenomenon. The amplitude amplification can be analyzed by employing Maximal Temporal Amplitude (MTA) which is a quantity measuring the maximum wave elevation at each spatial position during the observation time. Wavenumber value affects the extreme position of the wave but not the amplitude amplification. Meanwhile, modulational frequency value affects both terms. Comparison of the evolution of the BBM wave packet to the previous results obtained from KdV equation gives interesting outputs regarding the extreme position and the maximum wave peaking.
\end{abstract}

\section{Introduction}

This research is motivated by the need of generating extreme waves (also called freak, rogue, or giant waves) in a typical tank in a hydrodynamic laboratory. An extreme wave is defined as a wave which has significant height more than twice the height of measured wave train $[1,2]$. There are some physical mechanisms which cause the occurrence of the extreme wave. In [3], it was explained that it can occur due to wave-current interaction, geometrical or spatial focusing, focusing due to dispersion, focusing due to modulational instability, or soliton collision. An extreme wave can take place in deep water, shallow water, and at coastal area. It was reported that the wave appeared often at coastal area and in shallow water [4]. The extreme wave is a major threat to the ships and other marine structures because it can cause severe damage and loss when they are hit by the wave [5-11]. However, the occurrence of the extreme wave is unpredictable. The generation of an extreme wave in the laboratory is important to test the endurance of the ships or marine structures before they are installed and operated in the real environment. Many studies have been conducted to investigate the behavior of the extreme wave analytically and numerically [4, 12-28].

While ships and marine structures are typically built and operate on the offshore, the occurrence of the extreme wave in deep water is not as easily understood as the one in shallow water and coastal area since the wave on the surface of deep water may not be significantly affected by the topography and current. This fact has given a rise to the theory of modulational instability. The instability causes growing modulation of the wave envelope. The phenomenon happens as a wave nonlinearity behavior. This nonlinear event occurs when a wave is perturbed by other waves which cause envelope modulations that evolve into steep waves corresponding to nonlinear focusing of wave energy [3]. 
Modulational instability may be an appropriate mechanism to generate extreme waves in a wave tank. In a wave tank which initially contains still water, signals are given to the wavemakers generating waves to propagate downstream along the tank. Owing to the nonlinearity effect, the waves deform during their propagation (see [29-32]).

Extreme wave behavior due to modulational instability has been fiercely studied involving various wave equations. The evolution of Korteweg-de Vries (KdV) equation's solution in wave packet form was investigated in [33]. In the study, the $\mathrm{KdV}$ wave packet which is initially in the bichromatic signals experiences instability deforming the wave and causing an amplitude amplification. Bichromatic wave is formed from a superposition of two monochromatic waves which have same amplitudes and slightly different frequencies $[27,30]$. This result was obtained by deriving the solution of the $\mathrm{KdV}$ equation in the form of asymptotic series up to the third order. KdV's solution in higher order, up to the fifth order, was conducted in [34] and the result suggests that the higher the order is, the higher the amplitude amplification is. However, these two waves' maximum amplitudes are far lower than the amplitude of a wave generated by HUBRIS model although the extreme positions at which the maximum peaking happens conform. HUBRIS model is numerical wave model which was developed based on the Laplace equation (full water equation) [27]. Bichromatic signal deformation was also investigated by employing Boussinesq equation in [24]. The resulting wave give matching amplitude to the wave of HUBRIS model. Study on Benjamin-Bona-Mahony (BBM) model's wave packet with bichromatic signal is presented in [35]. The results suggest that the waves experience instability during its propagation. Moreover, if the frequencies in the bichromatic signal are closer to each other then the amplitude amplification tends to be higher; also higher initial amplitude may cause higher amplification on the amplitude.

Modulational instability also occurs when a monochromatic signal is perturbed by a pair of side bands of smaller amplitudes and slightly different frequencies. These waves form trichromatic wave also known as Benjamin-Feir type wave. Nonlinear evolution of wave packet with trichromatic signal based on $\mathrm{KdV}$ equation was studied in [18]. Investigating the wave packet evolution can also be carried out through the envelope evolution. In many studies, it has been derived that the envelope of the KdV wave packet is in the NLS equation form [36-40]. One of NLS' exact solutions, Soliton on Finite Background (SFB), shows an instability during its propagation. SFB itself is a best approximation to the Benjamin-Feir type wave. KdV wave packet with its NLS envelope has been applied to study the occurrence of extreme waves in many areas, not only in the fluid dynamic but also in the fields of plasma physics, quantum electronic, and optics [41-45]. BBM wave packet envelope had been also analyzed in [46]. The study found that envelope evolves satisfying the NLS equation.

BBM equation was firstly proposed as a revision of classic $\mathrm{KdV}$ equation which has dispersion relation contradicting to its physical wave behavior [47]. The classic KdV equation only works for small values of wavenumber. When the wavenumber values are large, the phase velocity of the KdV waves becomes negative, which indicates that the waves change their direction of propagation. This behavior is against the original assumption of forward-travelling waves. BBM equation yields more reasonable dispersion relation for any value of wavenumber. A family of $\mathrm{KdV}$ equation with exact dispersion relation was derived in [48]. The exact dispersion relation describes positive phase velocity for any value of wave number and this finding has corrected the shortcoming of the classic KdV. This exact $\mathrm{KdV}$ equation has been applied in some studies of extreme wave generation. BBM and $\mathrm{KdV}$ equations are originally derived from the same equation which is Laplace equation. These equations both describe shallow water wave propagation and yield soliton solution. However, the two equations in all likelihood illustrate waves with distinct characteristics.

In this study, we are interested in observing the evolution of BBM wave packet in the extreme wave context. The waves will be investigated through its envelope which is in NLS equation form. The SFB solution will be applied to analyze the instability which may trigger an extreme wave. The amplitude amplification will be measured through the waves' Maximal Temporal Amplitude (MTA), a quantity measuring the highest elevation at every spatial position in the domain during the observation time. It is also interesting to compare the results to the $\mathrm{KdV}$ equation waves which have been actively studied especially in extreme wave area.

In the next section, we will briefly review the BBM wave packet envelope which is adopted from the study in [46]. Also we will derive the elevation equation based on the wave packet and the obtained envelope equation. Section 3 contains explanation of two quantities which importantly describe the resulting wave characteristics, Amplitude Amplification Factor (AAF) and Maximal Temporal Amplitude (MTA). The resulting waves will be presented in Section 4 which is divided into two parts; the first part explains the modulational instability and phase singularity phenomenon and the second part presents the result comparison from BBM and $\mathrm{KdV}$ wave packet evolution. This paper is closed by presenting conclusions in Section 5 .

\section{Envelope Equation of BBM Wave Packet}

We consider propagating waves developed from regularized long wave equation, Benjamin-Bona-Mahony equation [47];

$$
\eta_{t}+\eta_{x}+\eta \eta_{x}-\eta_{x x t}=0
$$

where $\eta$ is wave elevation, $x$ is spatial variable and $t$ is time variable. The linear form of BBM equation (1) which describes dispersive effect to the equation yields dispersion relation stating the relation between the wavenumber $k$ and frequency $\omega$; the dispersion relation reads $\omega=k /\left(1+k^{2}\right)$. The BBM equation presented in (1) is in the nondimensional form with the scaling factors for the variables as well as the corresponding frequency and wavenumber are given as $\eta=$ $3 \tilde{\eta} / 2 h, x=\tilde{x} \sqrt{6} / h, t=\tilde{t} \sqrt{6 g / h}, \omega=\tilde{\omega} \sqrt{6 h / g / 6} / 6, k=$ $\tilde{k} h \sqrt{6} / 6$, where $\tilde{\eta}, \tilde{x}, \tilde{t}, \widetilde{\omega}, \widetilde{k}$ represent the laboratory/physical variables and $h$ and $g$ are water depth and gravitational acceleration, respectively. 
The deformation of the wave packet can be investigated through its envelope. An ansatz depicting the solution of (1) as a wave packet is taken,

$$
\eta(x, t)=A(\xi, \tau) e^{i(\omega t-k x)}+\text { c.c. }
$$

where $A(\xi, \tau)$ represents the envelope equation of the waves, $\xi$ and $\tau$ represent spatial and time variables, respectively, and c.c. denotes the complex conjugate. Envelope waves tend to move in slower velocity than the carrier waves. Therefore, $\xi$ and $\tau$ are called slow variables transformed from the fast variables $x$ and $t$ by the following relations,

$$
\begin{aligned}
\xi & =\varepsilon(x-p t), \\
\tau & =\varepsilon^{2} t, \\
p & =\frac{1+2 k \omega}{1+k^{2}},
\end{aligned}
$$

where $\varepsilon$ is a small real number. Through these fast-toslow variables transformation and applying the asymptotic expansion method, envelope equation $A(\xi, \tau)$ is derived and it is found that it evolves satisfying the so-called Nonlinear Schrodinger (NLS) equation

$$
A_{\xi}+i \beta A_{\tau \tau}+i \gamma|A|^{2} A=0
$$

where

$$
\begin{aligned}
& \beta=-\frac{1}{p^{3}}\left(2 \omega p+\frac{\omega^{2}}{k}\right), \\
& \gamma=\frac{\omega}{p}\left(\frac{2}{p-1}-\frac{k}{8 k^{2} \omega+2 \omega-2 k}\right) .
\end{aligned}
$$

Equation (4) is called spatial-NLS because it describes the change of wave envelope on every spatial position. The derivation of NLS equation from BBM equation can be thoroughly studied in [46].

NLS equation has many solutions. The second important solution is Soliton on Finite Background (SFB). This solution explains an interaction between a soliton and its background which have different frequencies along its propagation on the spatial domain [41]. SFB can also be represented as nonlinear interaction between a monochromatic signal perturbed by a pair of modulation waves having small frequency difference from the monochromatic's one $[40,41]$. The interaction triggers growing instability with a certain rate. The SFB reads $[41,49]$

$$
A(\xi, \tau)=A_{\mathrm{SFB}}(\xi, \tau) r_{0} e^{-i \hat{\gamma} r_{0}^{2} \xi},
$$

where

$$
\begin{aligned}
A_{\mathrm{SFB}}(\xi, \tau)= & \frac{u(\xi, \tau)+i v(\xi, \tau)}{\cosh (\sigma(\nu) \xi)-\sqrt{1-\left(\hat{\nu}^{2} / 2\right)} \cos (\nu \tau)} \\
u(\xi, \tau)= & \left(\hat{\nu}^{2}-1\right) \cosh (\sigma(\nu) \xi) \\
& +\sqrt{1-\frac{\widehat{v}^{2}}{2}} \cos (\nu \tau) \\
v(\xi, \tau)= & -\hat{v} \sqrt{2-\widehat{v}^{2}} \sinh (\sigma(\nu) \xi)
\end{aligned}
$$

and $\sigma(\nu)=\nu \sqrt{2 r_{0}^{2} \beta \gamma-\beta^{2} \nu^{2}}, \widehat{\nu}=\nu /\left(r_{0} \sqrt{\gamma / \beta}\right) ; \nu$ is modulation frequency. SFB reaches its maximum amplitude at $(0,2 n \pi / v), n \in \mathbb{Z}$ (see [50]). The frequency $\nu$ causes an amplitude amplification if the value in the interval $0<v<$ $r_{0} \sqrt{2 \gamma / \beta}$ (see [49]). It is convenient to rewrite the interval as $0<\hat{\nu}<\sqrt{2}$.

Let $A(\xi, \tau)$ describes the slowly varying envelope and satisfies the NLS equation (4); then the physical wave profile given by the surface elevation (2) can be written in real valued function

$$
\begin{aligned}
& \eta(x, t) \\
& =\frac{2 r_{0} \sqrt{u(\xi, \tau)^{2}+v(\xi, \tau)^{2}}}{\cosh (\sigma(\nu) \xi)-\sqrt{1-\left(\widehat{v}^{2} / 2\right)} \cos (\nu \tau)} \cos (\Phi(x, t)),
\end{aligned}
$$

where $\Phi(x, t)=\omega t-k x-\widehat{\gamma} r_{0}^{2} \xi+\arctan (v(\xi, \tau) / u(\xi, \tau))$ and $\xi$ and $\tau$ are defined as stated in the transformation (3).

\section{Amplitude Amplification and Maximal Temporal Amplitude}

The instability which happens to the SFB wave causes a growing amplitude during its propagation. Let $\Lambda$ be the maximum amplitude at an extreme position $(x, t)=(0,0)$; hence

$$
\begin{aligned}
\Lambda & =\eta(0,0) \\
& =\frac{2 r_{0} \sqrt{u(0,0)^{2}+v(0,0)^{2}}}{\cosh (0)-\sqrt{1-\left(\hat{v}^{2} / 2\right)} \cos (0)} \cos (\Phi(0,0)) .
\end{aligned}
$$

Since $v(0,0)=0$ and $\Phi(0,0)=0$, it is obtained that

$$
\Lambda=2 r_{0}\left(1+2 \sqrt{1-\frac{\hat{v}^{2}}{2}}\right) .
$$

In (10), $\Lambda$ is a decreasing function with respect to $\hat{v}$ and proportional to the initial amplitude $r_{0}$ at a far distance before the extreme position $x=0$. Through this relation a certain value for the maximum amplitude can be achieved by setting an appropriate value to parameter $r_{0}$.

Amplitude Amplification Factor (AAF) can be employed to measure the magnitude of the amplitude peaking. AAF is defined as the ratio of the maximum amplitude at the extreme position to the amplitude of the monochromatic wave at the far distance before it $[33,34,40]$. Therefore, we can state the $\mathrm{AAF}$ as

$$
\mathrm{AAF}=\frac{\Lambda}{2 r_{0}}=1+2 \sqrt{1-\frac{\widehat{v}^{2}}{2}}
$$

Equation (11) explains that $\mathrm{AAF} \rightarrow 1$ when $\widehat{v} \rightarrow \sqrt{2}$, meaning that the amplitude does not experience amplification. However, when $\widehat{v} \rightarrow 0$, the FAA $\rightarrow 3$; the amplitude of initially monochromatic wave will increase as much as three 


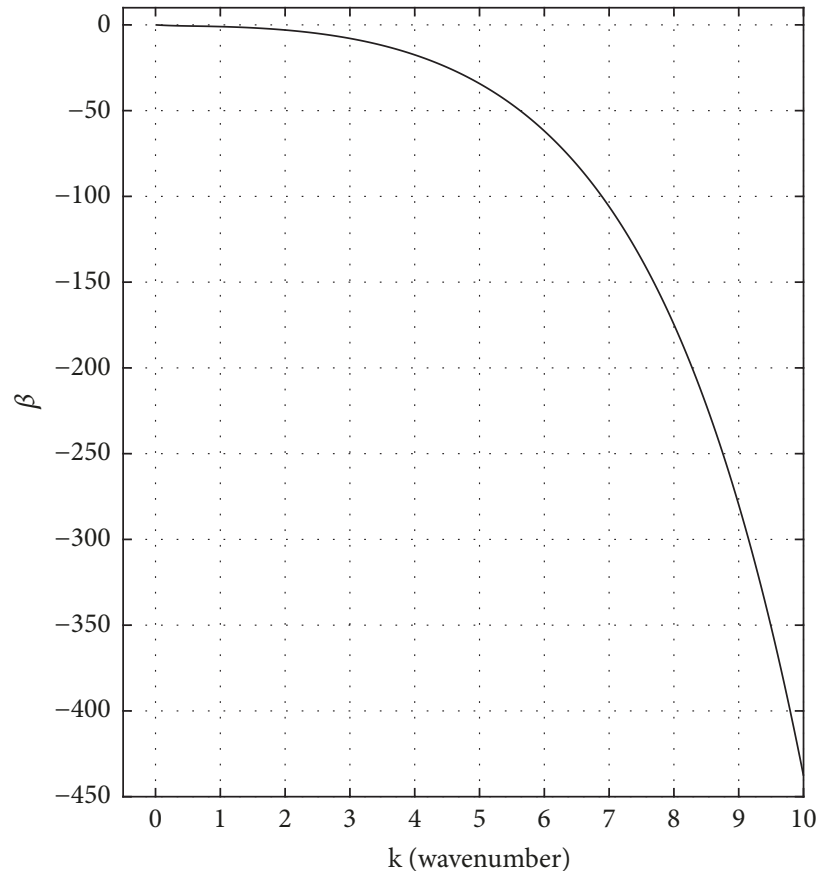

(a)

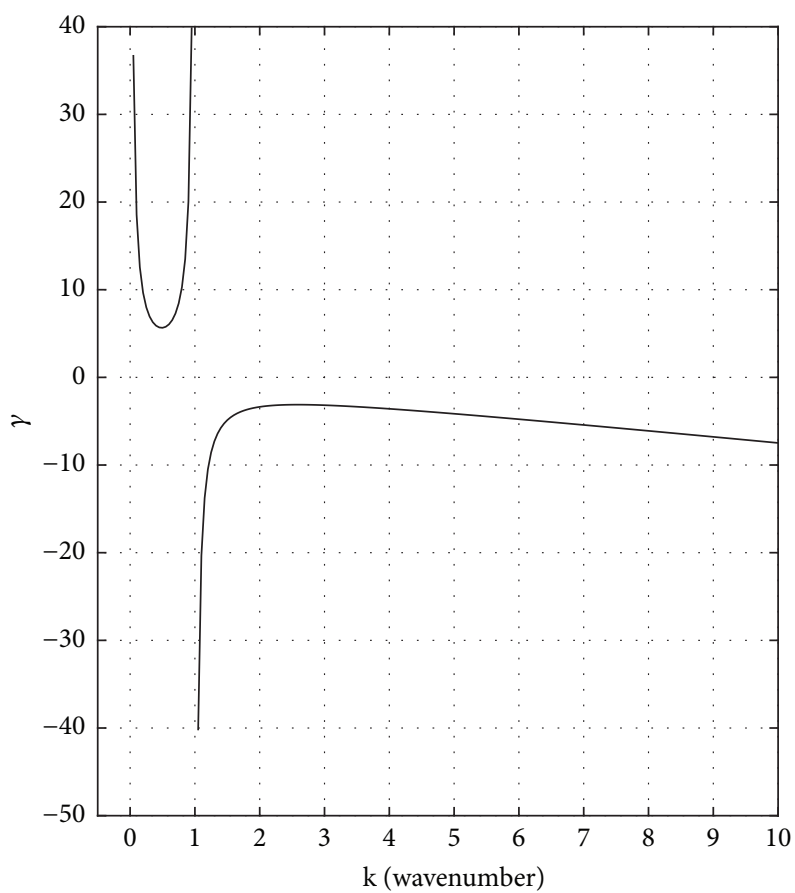

(b)

FIGURE 1: Graph of the value of dispersive coefficient $\beta$ (a) and nonlinear coefficient $\gamma$ (b) with respect to wave number $k$ from NLS equation (4).

times at the extreme position. This value also represents the maximum amplification the wave can achieve for any given initial amplitude $r_{0}$.

During the propagation, the wave elevation will change at every position. To investigate how the wave deforms along its propagation along the spatial domain, we apply a quantity called Maximal Temporal Amplitude (MTA). MTA measures the maximum elevation of the wave signal at every position $[24,40,50]$. MTA is defined as

$$
\operatorname{MTA}(x)=\max _{t} \eta(x, t) .
$$

MTA is invariant to time. This function can be applied to determine the amplitude amplification globally by comparing the maximum MTA with the MTA at the initial position of the wave.

\section{Results and Discussion}

4.1. Modulational Instability and Phase Singularity. Wave peaking occurs due to focusing phenomenon when waves experience modulational instability which is also known as Benjamin-Feir Instability [49]. For the NLS equation case, focusing can happen depending on the value of the coefficients of the dispersive and the nonlinear terms. If the values of both $\beta$ and $\gamma$ are of the same sign then both terms give balance effect resulting in the formation of solitary wave envelope [37]. Figure 1 depicts the graphs of $\beta$ and $\gamma$ with respect to the wavenumber $k$. For positive real valued $k$, the dispersive coefficient $\beta$ is a negative function while the nonlinear coefficient $\gamma$ is positive on $0<k<1$ and negative

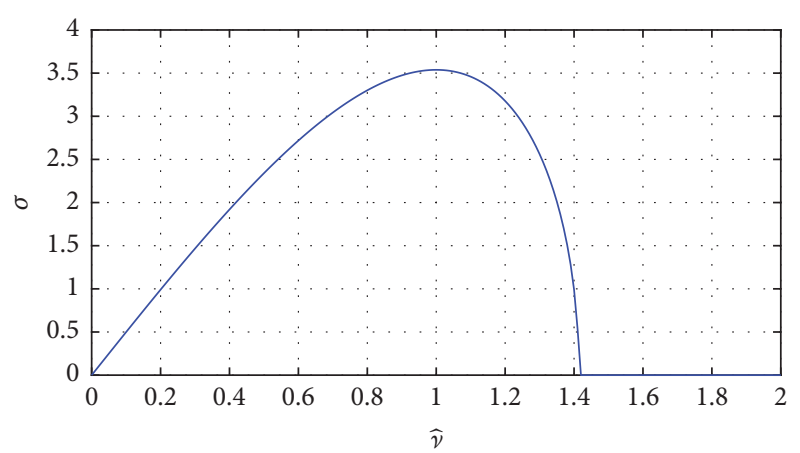

FIGURE 2: Graph of instability growth rate.

on $k>1$. Therefore, we can choose a value for $k$ in the interval $k>1$ which triggers the focusing phenomenon.

We investigate the wave propagation at water depth $h=$ $5 \mathrm{~m}$ and gravitational acceleration $g=9.8 \mathrm{~m} / \mathrm{s}^{2}$. Choosing $k=1.8751(\tilde{k}=0.9186 \mathrm{rad} / \mathrm{m})$ gives $\omega=0.4152(\widetilde{\omega}=$ $1.4238 \mathrm{rad} / \mathrm{s})$ through the dispersion relation, $\beta=-3.0964$ and $\gamma=-3.5380$. By setting $r_{0}=0,06\left(\widetilde{r}_{0}=0,2 \mathrm{~m}\right)$, we obtain the graph of the instability growth rate $\sigma$ over the value of $\widehat{v}$ as illustrated in Figure 2. It can be observed that the instability occurs only in the interval $0<\widehat{v}<\sqrt{2}$ and the highest instability happens when $\widehat{v}=1$. Figure 3 exhibits the wave profile $\eta(x, t)$ for $\widehat{v}=1$. It can be observed that amplitude amplification occurs at a certain period during the propagation. Moreover, the figure also exhibits phase singularity phenomenon which is indicated by 


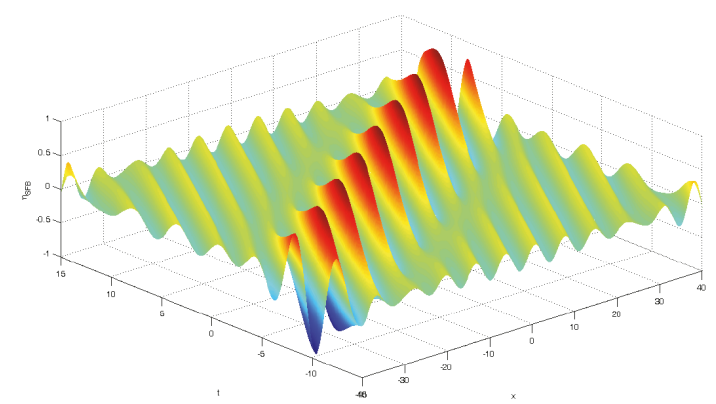

(a)

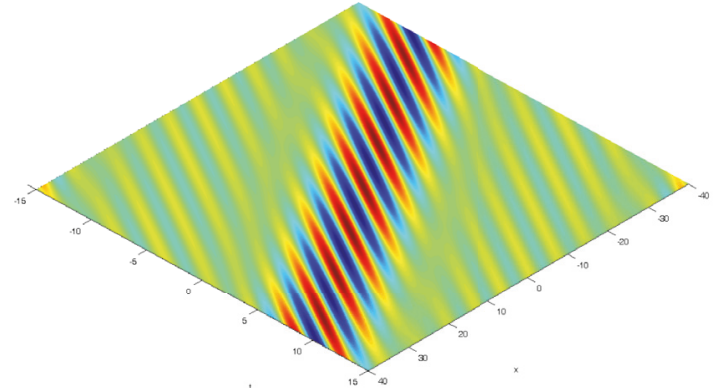

(b)

FIGURE 3: (a) Wave train profile and (b) wave contour of $\eta_{\mathrm{SFB}}(x, t)$ for $\widehat{\nu}=1$.

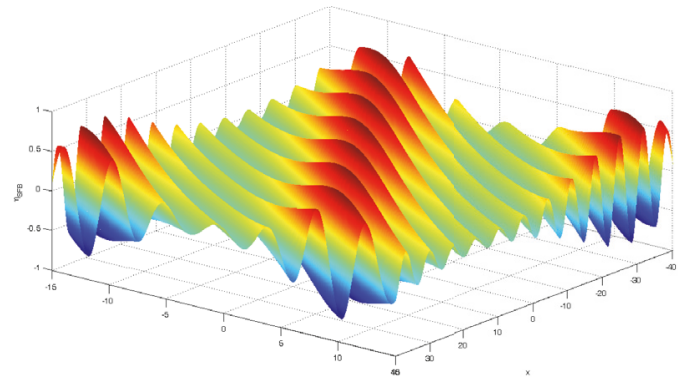

(a)

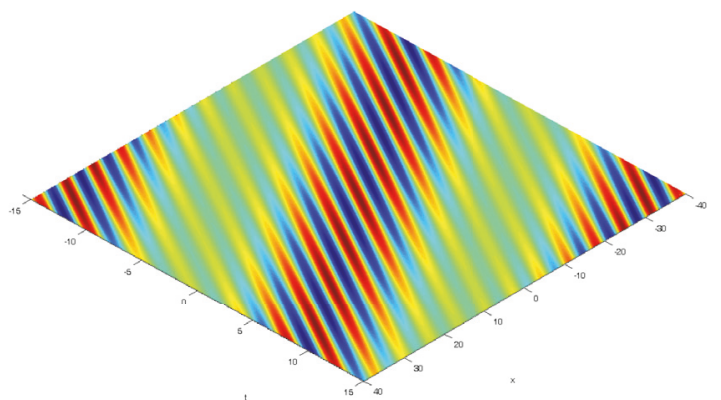

(b)

FIGURE 4: (a) Wave train profile and (b) wave contour of $\eta_{\mathrm{SFB}}(x, t)$ for $\widehat{\nu}=1.3$.

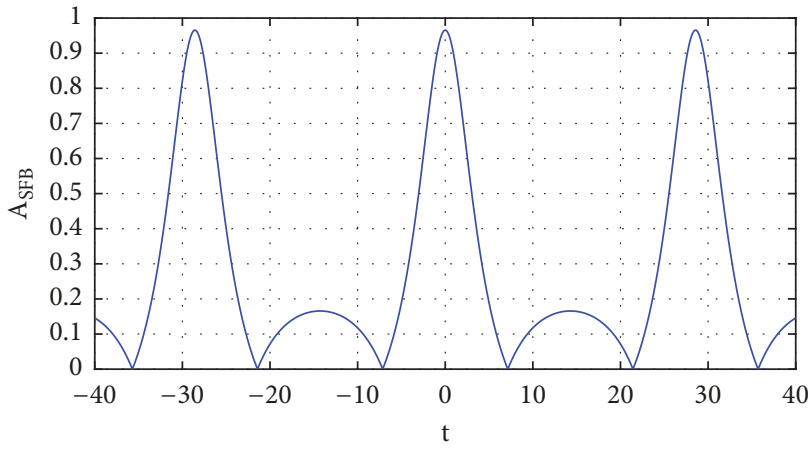

(a)

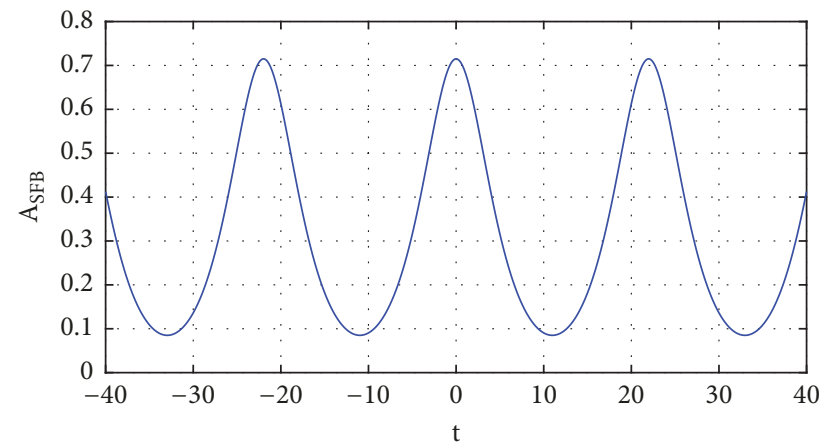

(b)

Figure 5: (a) Curve of envelope $\left|A_{\mathrm{SFB}}(x, t)\right|$ at $x=0$ for $\widehat{\nu}=1$. (b) Curve of envelope $\left|A_{\mathrm{SFB}}(x, t)\right|$ at $x=0$ for $\widehat{v}=1.3$.

wave dislocation when the waves split into two waves and merge into one wave near (before and after) the wave peaking. Phase singularity is a phenomenon which takes place when the real values of the amplitude $\left|A_{\mathrm{SFB}}\right|$ vanish $[40,50]$. This can be observed in Figure 5(a) in which the envelope curve intercepts the $x$ axis at some points. Wave dislocation is also known as optical vortices in the optical wave, line of darkness in the light wave, and thread of silence in the sound wave [51].

The wave dislocation also occurs when setting $\widehat{v}=0.7$, but it does not when $\widehat{v}=1.3$, or when $\widehat{v}=1.4$. Figure 4 displays the wave train and contour when $\widehat{v}=1.3$. This indicates that not all values of $\hat{v}$ in the interval $(0, \sqrt{2})$ trigger the phase singularity; there is a value in $(0, \sqrt{2})$ which is a threshold of the event to occur. We call this value as critical point and it had been determined that $\widehat{v}_{\text {crit }}=\sqrt{3 / 2}$ as presented in $[40,49]$. Hence, the phase singularity occurs when the value of $\hat{v}$ is chosen in the interval $(0, \sqrt{3 / 2})$. Figure 5 (a) illustrates the phase singularity when $\hat{v}=1$ as the curve of the envelope at the extreme position $x=0$ intercepts the horizontal axis, $\left|A_{\mathrm{SFB}}(0, \tau)\right| \geq 0$. Meanwhile, Figure 5(b) displays the envelope curve when $\widehat{v}=1.3$; the curve is definitely positive.

Spatial SFB is symmetrical to the time variable $t$ and in a soliton form symmetrical to line $x=0$. Figure 6 displays the wave signals at some spatial points and the symmetrical behavior. At a far distance before the extreme position, the SFB signal is monochromatic waves with amplitude $2 r_{0}$ and 

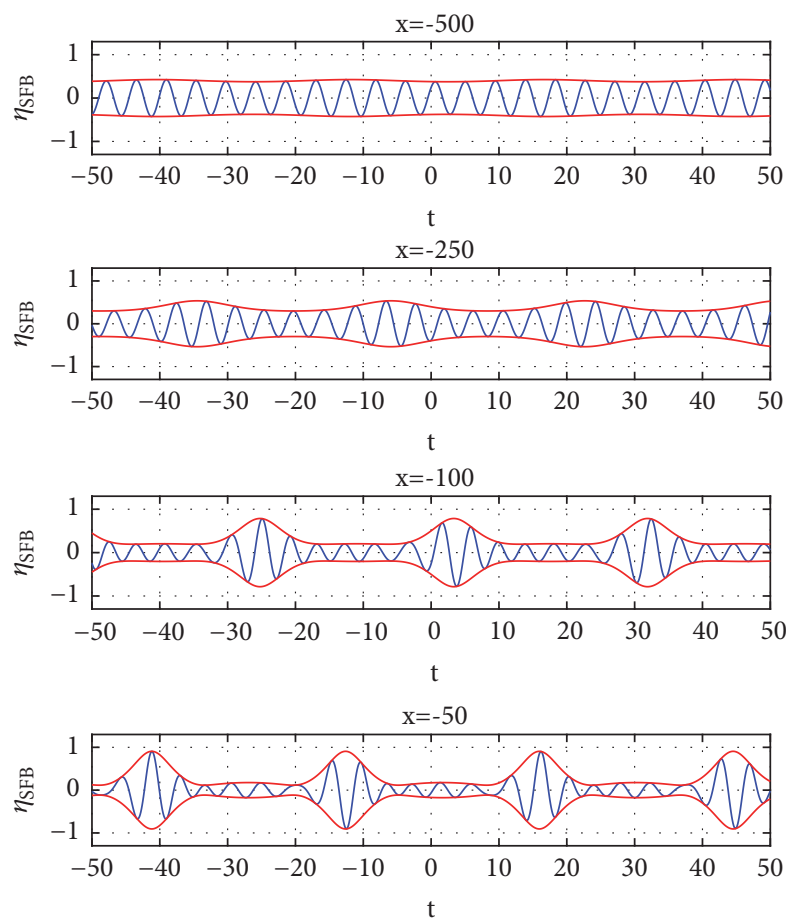

Figure 6: Wave signal $\eta_{\mathrm{SFB}}(x, t)$ (blue) and envelope $\left|A_{\mathrm{SFB}}\right|\left(\right.$ red) at some spatial position for $\left(\widetilde{r_{0}} ; \widetilde{k} ; \widehat{\nu}\right)=(0.2 ; 0.9186 ; 1)$.
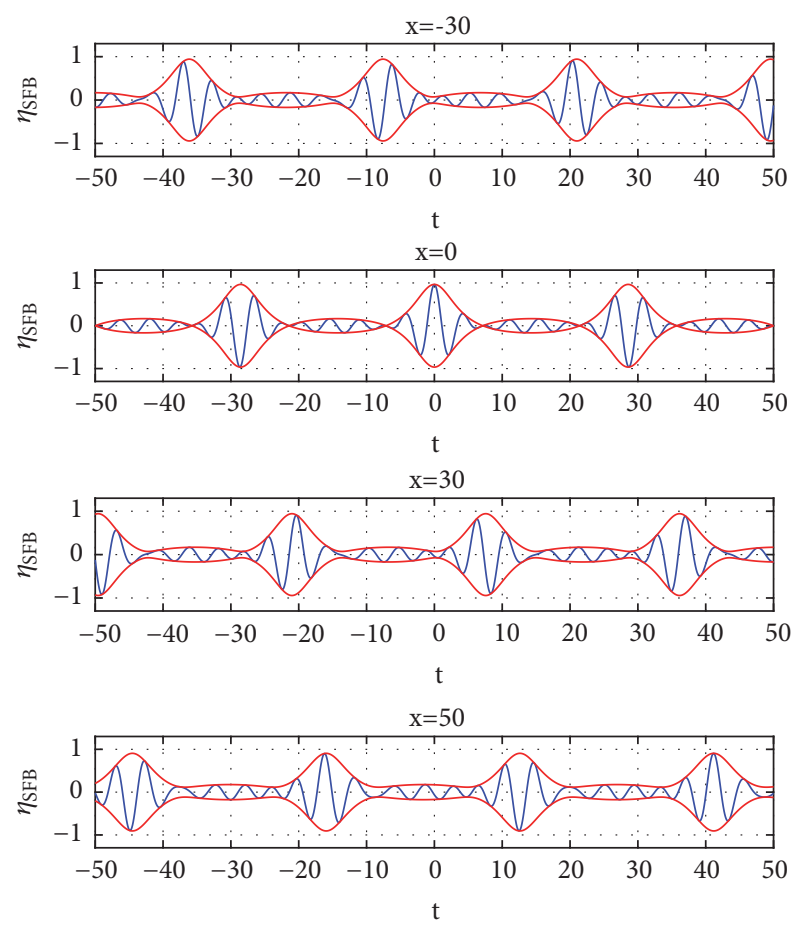

frequency $\omega$. The instability is taking place at position $x=$ $-500 \mathrm{~m}$ and the waves start to deform. It gets obvious as the waves propagate reaching position $x=-250 \mathrm{~m}$. The instability is indicated by the growing modulation envelope. The amplitude keeps increasing as it reaches $x=-100 \mathrm{~m}$, $x=-50 \mathrm{~m}$, and $x=-30 \mathrm{~m}$ until it reaches the maximum peaking at $x=0$. It can be observed that new modulations emerge between the high envelope and it becomes more obvious at $x=0$. Furthermore, the phase singularity can be remarked when $x=0$; the peak or the trough are on the $t$ axis. Thereafter, the amplitude starts to decrease symmetrically.

Figure 7 exhibits the amplitude spectra of the SFB signal from the Figure 6 . At $x=-500 \mathrm{~m}$, the wave which is initially a monochromatic signal with frequency $\omega=1.4238 \mathrm{rad} / \mathrm{s}$ is being perturbed by a new pair of modulation (side bands) with frequency difference $0.2199 \mathrm{rad} / \mathrm{s}$. In this state, the forming wave is trichromatic wave. Furthermore, the perturbing wave amplitude is increasing and new other side bands appear as the waves propagate along the $x$ axis until it reaches the maximum position $x=0$ and the nearest side bands surpass the monochromatic signal amplitude. This phenomenon explains the modulational instability which occurs during the wave propagation. Moreover, the occurrence of the new modulations near the initially monochromatic signal suggests a self-focusing phenomenon which is energy transfer among the surrounding waves.

Figure 8 displays the MTA and spatial evolution of (8). The graphs have been shifted such that the wave's maximum peaking occurs on the positive $x$ axis and the initially monochromatic wave appears at $x=0$. In terms of generating wave in a hydrodynamic laboratory, this shifted spatial value is more relatable. In the figure, it can be observed that the wave propagates toward the positive $x$ direction and reaches the maximum peaking at $x=777.6 \mathrm{~m}$. Far before the extreme position, the wave is being modulated and its amplitude keeps increasing in significant manner. This result is clearly displayed by the MTA which is increasingly monotone until it reaches $x=777.6 \mathrm{~m}$. The wave is initially at amplitude $0.4 \mathrm{~m}$ and reaches its maximum value at amplitude $0,9657 \mathrm{~m}$, meaning that it is 2.4142 times higher than the initial amplitude. After reaching the maximum peaking, the amplitude starts decreasing and forming symmetrical value to the extreme position axis.

Different values of wavenumber affect the extreme position but may not affect the maximum peaking. These results are exhibited in Figure 9(a). If the wavenumber is higher then the extreme position is farther. This output happens for chosen wavenumbers $\tilde{k}=0.6 \mathrm{rad} / \mathrm{m}, \widetilde{k}=0.7 \mathrm{rad} / \mathrm{m}$ and $\widetilde{k}=0.9186 \mathrm{rad} / \mathrm{m}$ with respective extreme position $x=290.6 \mathrm{~m}, x=500.5 \mathrm{~m}$, and $x=777.6 \mathrm{~m}$. However, when the wavenumber is set to higher value $\widetilde{k}=3 \mathrm{rad} / \mathrm{m}$, the extreme position becomes closer at $x=564.6 \mathrm{~m}$ to the $x=0$ compared to the one with wavenumber $\widetilde{k}=$ $0.9186 \mathrm{rad} / \mathrm{m}$. These findings may lead to some hypotheses regarding the extreme position; however further study needs to be conducted.

The change in the value of $\hat{v}$ may affect the maximum peaking. Figure 9(b) exhibits the MTAs for some values of $\widehat{\nu}$. The maximum MTAs for $\widehat{v}=0.2, \widehat{v}=0.5, \widehat{v}=0.7$ and $\widehat{v}=1$ are, respectively, $1.1913 \mathrm{~m}, 1.1482 \mathrm{~m}, 1.0949 \mathrm{~m}$, and $0.9656 \mathrm{~m}$. The smaller the value of $\widehat{v}$ is, the higher the maximum peaking will be. This output resonates the relation between $\widehat{v}$ an AAF 

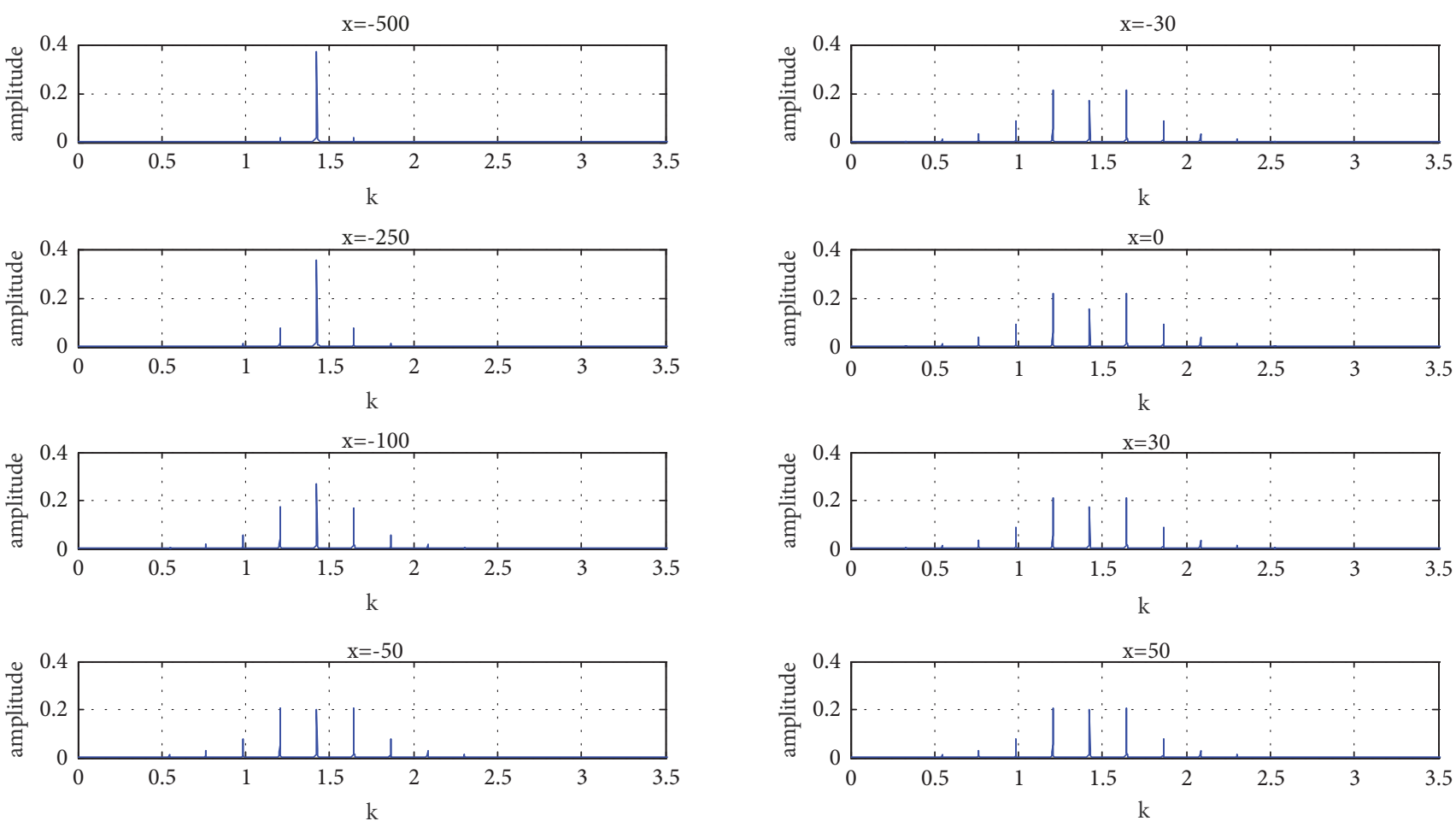

FIGURE 7: Amplitude spectra of wave signal $\eta_{\mathrm{SFB}}(x, t)$ at some spatial positions for $\left(\widetilde{r_{0}} ; \tilde{k} ; \widehat{v}\right)=(0.2 ; 0.9186 ; 1)$.

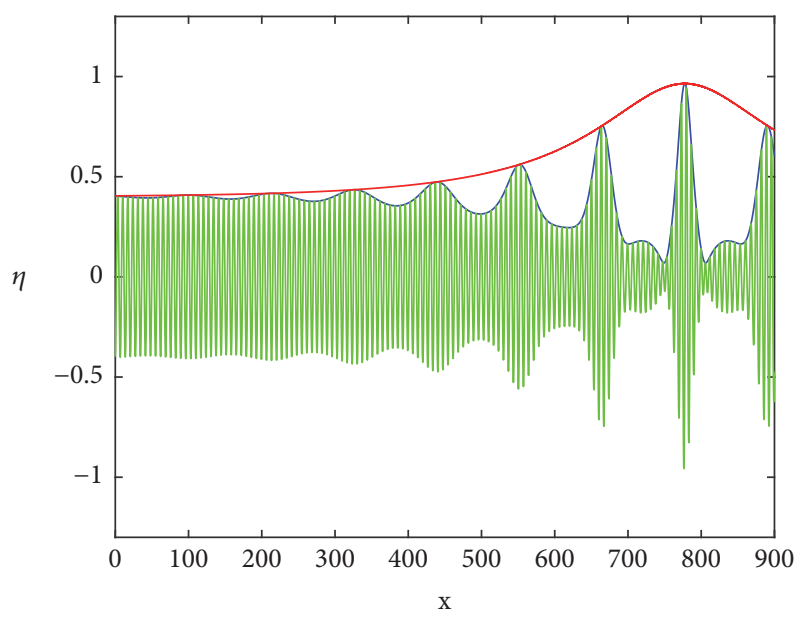

FIGURE 8: Elevation $\eta_{\mathrm{SFB}}(x, t)$ (green), envelope $\left|A_{\mathrm{SFB}}\right|$ (blue), and MTA (red) of the BBM wave packet for $\left(\widetilde{r}_{0} ; \widetilde{k} ; \widehat{v}\right)=(0.2 ; 0.9186 ; 1)$.

stated in (11). Hence the AAF can be computed analytically, which are 2.9799, 2.8708, 2.7378, and 2.4142 for $\hat{v}=0.2$, $\widehat{v}=0.5, \widehat{v}=0.7$, and $\widehat{v}=1$, respectively. Moreover, besides affecting the AAF, the value of $\widehat{v}$ also affects the extreme position. It can be observed from Figure 9(b) that as the AAF is getting higher, the extreme position may become farther from the origin.

4.2. Comparison of BBM and KdV Wave Packet Evolution. Previous researches on wave envelope evolution had been conducted by employing the Korteweg-de Vries (KdV) equation. Some equations in $\mathrm{KdV}$ equation family had been proposed and the dynamic in its propagation as a wave packet had been also studied [40-45, 49, 50]. In [37], NLS equation was derived from the classic $\mathrm{KdV}$ equation introduced by Korteweg and De Vries in 1895 and it was obtained that the NLS equation is of the defocusing type at which the dispersive and nonlinear terms are always in different signs. Hence, the both terms act to widen the envelope and envelope solitary waves are unlikely to occur. However, NLS equation derived from the exact $\mathrm{KdV}$ equation which was proposed by Van Groesen in 1998 [48] may yield focusing solution. The NLS envelope of the KdV wave packet can be observed in $[33,40,49]$. There is a critical wavenumber for which the dispersive and nonlinear terms of the NLS equation are in the same sign, which is $k>0.23$. Hence, choosing the value of $k$ in the interval $k>0.23$ gives focusing type NLS. For the KdVNLS equation, the modulation frequency $\widehat{v}$ which triggers the instability lies in the interval $0<\hat{v}<\sqrt{2}$ and the critical modulation frequency for which the phase singularity may occur is $\widehat{\nu}_{\text {crit }}=\sqrt{3 / 2}$. The maximum AAF approaches 3 as the $\widehat{v}$ approaches 0 . The characteristics of the exact KdV-NLS and the BBM-NLS that we analyze in this study are pretty much similar. Therefore, here we are concerned with comparing both results. The evolution of the wave profile, envelope, and MTA of the exact KdV wave packet is presented in Figure 10.

The difference between the BBM-NLS and KdV-NLS is sited on the coefficients of the dispersive $(\beta)$ and nonlinear $(\gamma)$ terms. Therefore, the coefficients' values may affect the resulting wave and its evolution for an assigned wavenumber 


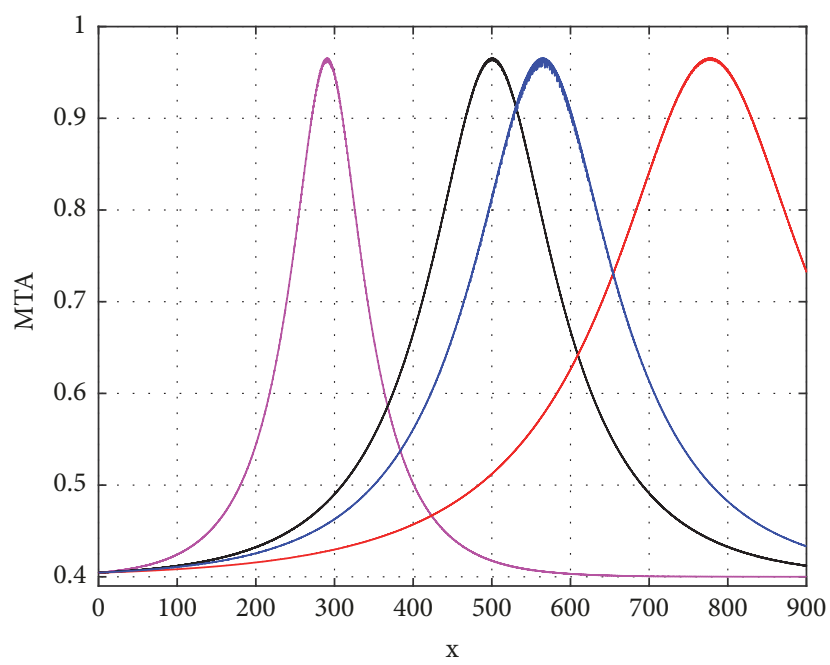

(a)

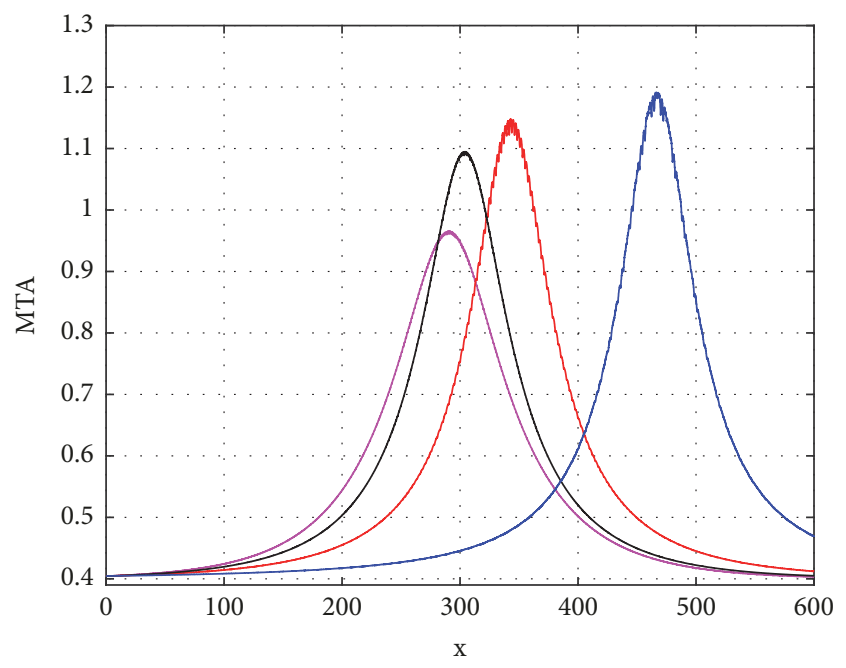

(b)

Figure 9: (a) BBM's MTAs for some values of wavenumbers: $\widetilde{k}=0.6$ (magenta), $\widetilde{k}=0.7$ (black), $\widetilde{k}=0.9186$ (red), and $\widetilde{k}=3$ (blue) with $\left(\widetilde{r}_{0} ; \widehat{v}\right)=(0.2 ; 1)$. (b) BBM's MTAs for some values of modulation frequencies $\widehat{v}=1$ (magenta), $\widehat{v}=0.7$ (black), $\widehat{v}=0.5$ (red), and $\widehat{v}=0.2$ (blue) with $\left(\widetilde{r}_{0} ; \widetilde{k}\right)=(0.2 ; 0.6)$.

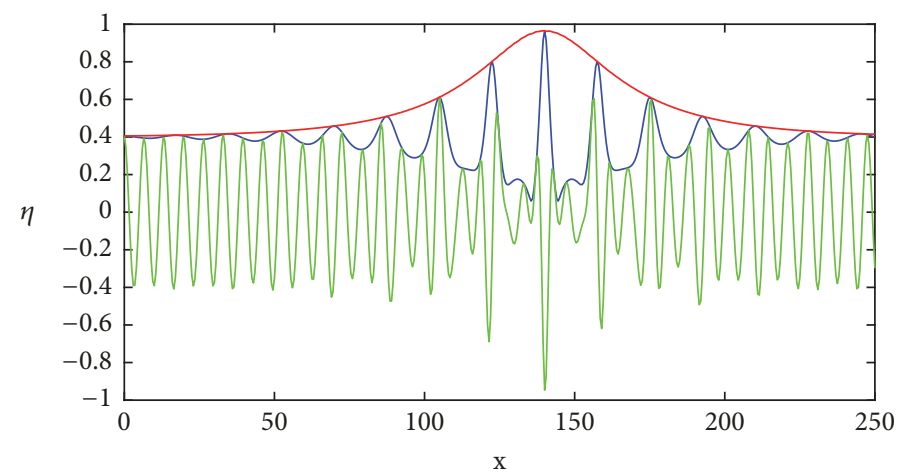

FigURE 10: Elevation $\eta_{\mathrm{SFB}}(x, t)$ (green), envelope $\left|A_{\mathrm{SFB}}\right|$ (blue), and MTA (red) of the KdV wave packet for $\left(\widetilde{r_{0}} ; \widetilde{k} ; \widehat{\nu}\right)=(0.2 ; 0.9186 ; 1)$.

$k$. Besides the wave profile appears to be different in terms of density of the carrier waves as we compare wave profile in Figure 8 to the one in Figure 10, the extreme positions are also significantly dissimilar. Figure 11 exhibits the MTA of $\mathrm{KdV}$ and $\mathrm{BBM}$ wave packet propagation for same chosen values $\left(\widetilde{r}_{0} ; \widetilde{k} ; \widehat{v}\right)=(0.2 ; 0.9186 ; 1)$. From Figure 11 , it can be observed that the BBM waves need farther distance to achieve its extreme position where the maximum peaking takes place compared to the $\mathrm{KdV}$ waves although the magnitudes of the maximum peaking are the same. The extreme position of $\mathrm{BBM}$ waves is at $x=777.6 \mathrm{~m}$ while that of the $\mathrm{KdV}$ waves is at $x=140 \mathrm{~m}$. Moreover, the KdV's MTA appears to be steeper than the BBM's MTA. These results may indicate that $\mathrm{BBM}$ wave packet deforms in slower manner while $\mathrm{KdV}$ wave packet tends to deform in faster way. However, when a smaller value for $\tilde{k}$ is given, the extreme position of the $\mathrm{BBM}$ waves is closer to the origin than the $\mathrm{KdV}$ waves as exhibited in Figure 12. BBM waves' extreme position is at $x=290.6 \mathrm{~m}$ whereas KdV waves' extreme position is at $x=$ $427 \mathrm{~m}$.

\section{Conclusion}

The dynamic of BBM wave packet propagation can be observed through the evolution of its envelope. It has been derived that the envelope evolves satisfying the NLS equation. We have shown that the NLS equation is of focusing type for wavenumber $k$ value lying in the interval $k>1$. Through one NLS' exact solution, Soliton on Finite Background, modulational instability may happen to the waves during the propagation which lead to amplitude amplification. The instability may occur when the value of modulation frequency $\hat{v}$ is one in the interval $(0, \sqrt{2})$. However, phase singularity which is a phenomenon indicating the occurrence of extreme wave may take place if $\widehat{v}$ is a value in interval $(0, \sqrt{3 / 2})$. The maximum amplitude amplification factor (AAF) approaches 3 as the $\widehat{v}$ approaches 0 . Furthermore, the extreme position at which the maximum peaking occurs can be observed through the waves' MTA. The extreme position may vary depending on the value of the wavenumber $k$ while the maximum peaking is retained. However, further study is needed to analyze how 


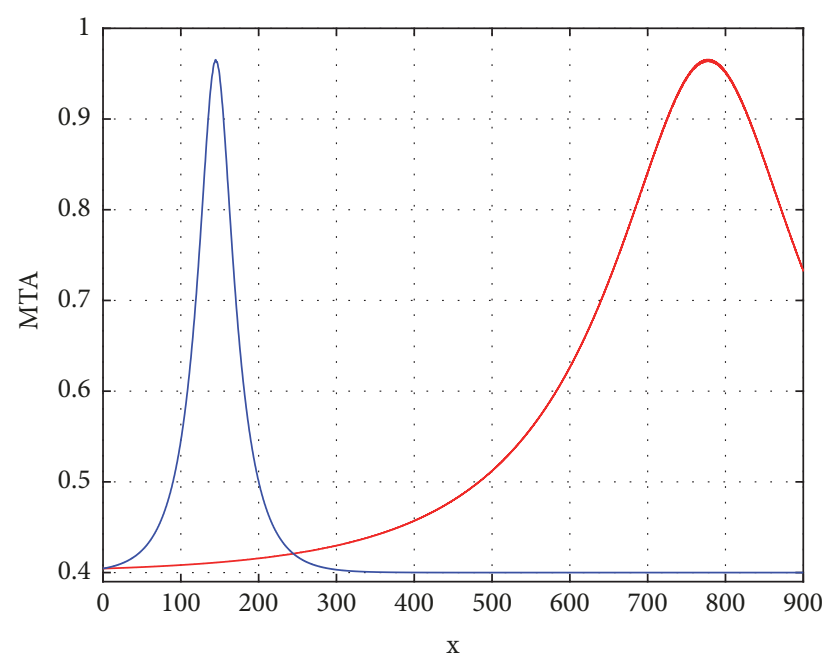

Figure 11: BBM's MTA (red) and KdV's MTA (blue) for $\left(\widetilde{r}_{0} ; \widetilde{k} ; \widehat{v}\right)=$ $(0.2 ; 0.9186 ; 1)$.

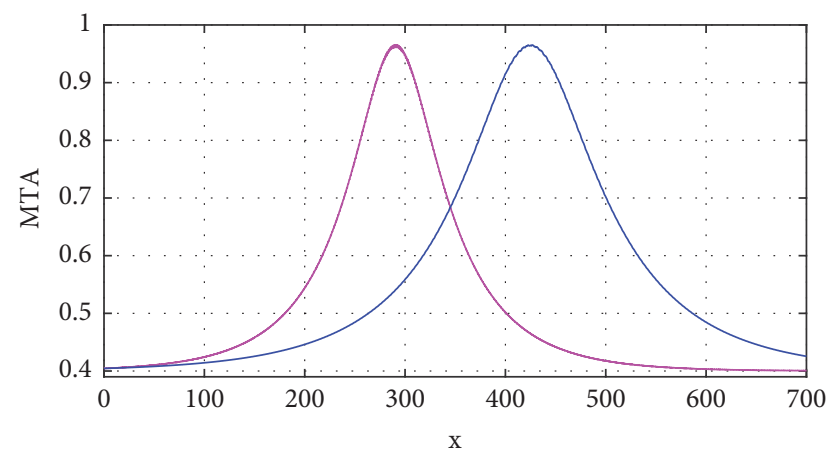

Figure 12: BBM's MTA (red) and KdV's MTA (blue) for $\left(\widetilde{r}_{0} ; \widetilde{k} ; \widehat{v}\right)=$ $(0.2 ; 0.6 ; 1)$.

the wavenumber may affect the extreme position. Modulation frequency $\hat{v}$ also affects the extreme position as well as the value of maximum peaking which has been proven through the AAF equation. We have also observed that BBM-NLS is similar to the KdV-NLS. Nevertheless, both equations give different extreme positions even though the magnitudes of the maximum peaking are the same.

\section{Data Availability}

No data were used to support this study.

\section{Conflicts of Interest}

The authors declare that there are no conflicts of interest regarding the publication of this paper.

\section{Acknowledgments}

This research is funded by Penelitian Profesor, Syiah Kuala University, 2018, with Contract no. 34/UN11.2/PP/PNBP/ SP3/2018 and Penelitian Tim Pascasarjana, 2018, with Contract no. 115/UN11.2/PP/SP3/2018.

\section{References}

[1] R. G. Dean, Freak Waves: A Possible Explanation, Water Wave Kinetics, Kluwer, pp. 609-621, Amsterdam, Netherlands, 1990.

[2] S. P. Kjeldsen, "Dangerous wave groups," Norwegian Maritime Research, vol. 12, no. 2, pp. 4-16, 1984.

[3] C. Kharif, E. Pelinovsky, and A. Slunyaev, Rogue Waves in the Ocean, Observation, Theories and Modeling, Springer, New, 2009.

[4] I. Nikolkina and I. Didenkulova, "Catalogue of rogue waves reported in media in 2006-2010," Natural Hazards, vol. 61, no. 3, pp. 989-1006, 2012.

[5] M. D. Earle, "Extreme wave conditions during Hurricane Camille," Journal of Geophysical Research: Atmospheres, vol. 80, no. 3, pp. 377-379, 1975.

[6] N. Mori and P. C. Liu, "Analysis of freak wave measurements in the Sea of Japan," Ocean Engineering, vol. 29, no. 11, pp. 13991414, 2002.

[7] B. V. Divinsky, B. V. Levin, L. I. Lopatikin, E. N. Pelinovsky, and A. V. Slyungaev, "A freak wave in the Black Sea, observations and simulation," Doklady Earth Sciences, vol. 395, pp. 438-443, 2004.

[8] K. Trulsen and K. Dysthe, "Freak waves a three dimensional wave simulation," in Proceedings of of the 21st Symposium on Naval Hydrodynamics, E. P. Rood, Ed., pp. 550-558, National Academy Press, 1997.

[9] R. Smith, "Giant waves," Journal of Fluid Mechanics, vol. 77, no. 3, pp. 417-431, 1976.

[10] A. Toffoli, "Extreme and Rogue Waves in Directional Wave Fields," The Open Ocean Engineering Journa, vol. 4, no. 1, pp. 24-33, 2011.

[11] T. Waseda, H. Tamura, and T. Kinoshita, "Freakish sea index and sea states during ship accidents," Journal of Marine Science and Technology (Japan), vol. 17, no. 3, pp. 305-314, 2012.

[12] T. Waseda, M. Sinchi, K. Kiyomatsu et al., "Deep water observations of extreme waves with moored and free GPS buoys," Ocean Dynamics, vol. 64, no. 9, pp. 1269-1280, 2014.

[13] Z. Hu, W. Tang, H. Xue, and X. Zhang, "Numerical study of rogue waves as nonlinear Schrodinger breather solutions under finite water depth," Wave Motion, vol. 52, pp. 81-90, 2015.

[14] A. Islas and C. M. Schober, "Rogue waves, dissipation, and downshifting," Physica D: Nonlinear Phenomena, vol. 240, no. 12, pp. 1041-1054, 2011.

[15] Z. Xu, H. Chen, and Z. Da, "Rogue wave for the $(2+1)-$ dimensional Kadomtsev-Petviashvili equation," Applied Mathematics Letters, vol. 37, pp. 34-38, 2014.

[16] A. Slunyaev, A. Sergeeva, and E. Pelinovsky, "Wave amplification in the framework of forced nonlinear Schrodinger equation: the rogue wave context," Physica D: Nonlinear Phenomena, vol. 303, pp. 18-27, 2015.

[17] E. Cahyono, L. O. Ngkoimani, and M. Ramli, "Notes on multiparameters perturbation method for dispersive and nonlinear partial differential equations," International Journal of Mathematical Analysis, vol. 9, pp. 2121-2132, 2015.

[18] M. Ramli, "Nonlinear evolution of wave group with three frequencies," Far East Journal of Mathematical Sciences, vol. 97, no. 8, pp. 925-937, 2015.

[19] S. Wabnitz, C. Finot, J. Fatome, and G. Millot, "Shallow water rogue wavetrains in nonlinear optical fibers," Physics Letters A, vol. 377, no. 12, pp. 932-939, 2013. 
[20] R. Peric, N. Hoffmann, and A. Chabchoub, "Initial wave breaking dynamics of Peregrine-type rogue waves: a numerical and experimental study," European Journal of Mechanics - B/Fluids, vol. 49, no. part A, pp. 71-76, 2015.

[21] J. M. Blackledge, "A generalized nonlinear model for the evolution of low frequency freak waves," IAENG International Journal of Applied Mathematics, vol. 41, no. 1, pp. 33-55, 2011.

[22] M. Onorato, S. Residori, U. Bortolozzo, A. Montina, and F. T. Arecchi, "Rogue waves and their generating mechanisms in different physical contexts," Physics Reports, vol. 528, no. 2, pp. 47-89, 2013.

[23] H. Fernández, V. Sriram, S. Schimmels, and H. Oumeraci, "Extreme wave generation using self correcting method revisited," Coastal Engineering Journal, vol. 93, pp. 15-31, 2014.

[24] M. Ramli, "Amplitude amplification factor of bi-chromatic waves propagation in hydrodynamic laboratories," IAENG International Journal of Applied Mathematics, vol. 46, no. 1, pp. 29-34, 2016.

[25] Marwan and Andonowati, "Wave deformation on the propagation of bi-chromatics signal and its effect to the maximum amplitude," Journal of Mathematical Sciences, vol. 8, pp. 81-87, 2003.

[26] Andonowati and E. Van Groesen, "Optical pulse deformation in second order nonlinear media," Journal of Nonlinear Optical Physics \& Materials, vol. 12, no. 2, pp. 221-234, 2003.

[27] J. Westhuis, E. Van Groesen, and R. Huijsmans, "Experiments and numeric of bichromatic wave group," Journal of Waterway, Port, Coastal, and Ocean Engineering, vol. 127, no. 6, pp. 334342, 2001.

[28] X.-Z. Zhao, C.-H. Hu, and Z.-C. Sun, "Numerical simulation of extreme wave generation using VOF method," Journal of Hydrodynamics, vol. 22, no. 4, pp. 466-477, 2010.

[29] E. van Groesen and E. S. Andonowati, "Nonlinear effects in bichromatic surface waves," in Proceedings of the Estonian Academy of Sciences Physics, vol. 48, pp. 206-229, 1999.

[30] C. T. Stansberg, "On the non linear behaviour of ocean wave groups, Ocean Wave Measurement and Analysis," Society of Civil Engineers (ASCE), vol. 2, pp. 1127-1241, 1998.

[31] J. Westhuis, E. van Groesen, and R. Huijsmans, "Long time evolution of unstable bi-chromatic waves," in Proceedings of the 15th IWWW and FB, pp. 184-187, Caesarea Israel, 2000.

[32] J. Westhuis, E. Van Groesen, and R. Huijsmans, "Experiments and numerics of bi-chromatic wave groups," Journal of Waterway, Port, Coastal, and Ocean Engineering, vol. 127, no. 6, pp. 334-342, 2000.

[33] Marwan, "On the maximal temporal amplitude of down stream running nonlinear water waves," Tamkang Journal of Mathematics, vol. 41, no. 1, pp. 51-69, 2010.

[34] M. Ramli, S. Munzir, T. Khairuman, and V. Halfiani, "Amplitude increasing formula of bichromatic wave propagation based on fifth order side band solution of Korteweg de Vries equation," Far East Journal of Mathematical Sciences, vol. 93, no. 1, pp. 97117, 2014.

[35] V. Halfiani and M. Ramli, "Deformation of bichromatic wave groups based on third order side band solution of BenjaminBona-Mahony equation," Journal of Physics: Conference Series, vol. 890, no. 1, Article ID 012011, 2017.

[36] M. J. Ablowitz, Nonlinear dispersive waves, Cambridge Texts in Applied Mathematics, Cambridge University Press, New York, 2011.
[37] J. P. Boyd and G.-Y. Chen, "Weakly nonlinear wavepackets in the Korteweg-de Vries equation: the KDV/NLS connection," Mathematics and Computers in Simulation, vol. 55, no. 4-6, pp. 317-328, 2001.

[38] M. Chirilus-Bruckner, W.-P. Düll, and G. Schneider, "Validity of the KdV equation for the modulation of periodic traveling waves in the NLS equation," Journal of Mathematical Analysis and Applications, vol. 414, no. 1, pp. 166-175, 2014.

[39] G. Schneider, "Justification of the NLS approximation for the $\mathrm{KdV}$ equation using the Miura transformation," Advances in Mathematical Physics, Article ID 854719, 2011.

[40] W. M. Kusumawinahyu, on The Onset of Wave Breaking [Ph.D. thesis], Institut Teknologi Bandung, Bandung, Indonesia, 2006.

[41] N. N. Akhmediev and N. Ankiewicz, Solitons: Nonlinear Pulses and Beams, Chapman and Hall, London, 1997.

[42] S. K. El-Labany, W. M. Moslem, N. A. El-Bedwehy, R. Sabry, and H. N. Abd El-Razek, "Rogue wave in Titan's atmosphere," Astrophysics and Space Science, vol. 338, no. 1, pp. 3-8, 2012.

[43] M. Bacha, S. Boukhalfa, and M. Tribeche, "Ion-acoustic rogue waves in a plasma with a q-nonextensive electron velocity distribution," Astrophysics and Space Science, vol. 341, no. 2, pp. 591-595, 2012.

[44] A.-U. Rahman and S. Ali, "Solitary and rogue waves in FermiDirac plasmas: Relativistic degeneracy effects," Astrophysics and Space Science, vol. 351, no. 1, pp. 165-172, 2014.

[45] S. A. El-Wakil, E. M. Abulwafa, A. Elhanbaly, and E. K. ElShewy, "Rogue waves for Kadomstev-Petviashvili equation in electron-positron-ion plasma," Astrophysics and Space Science, vol. 353, no. 2, pp. 501-506, 2014.

[46] V. Halfiani, Salmawaty, and M. Ramli, "An envelope equation of Benjamin-Bona-Mahony wave group," Far East Journal of Mathematical Sciences, vol. 102, no. 6, Article ID A2049, pp. 1077-1090, 2017.

[47] T. B. Benjamin, J. L. Bona, and J. J. Mahony, "Model equations for long waves in nonlinear dispersive systems," Philosophical Transactions of the Royal Society A: Mathematical, Physical \& Engineering Sciences, vol. 272, no. 1220, pp. 47-78, 1972.

[48] E. Van Groesen, "Wave groups in uni-directional surface-wave models," Journal of Engineering Mathematics, vol. 34, no. 1-2, pp. 215-226, 1998.

[49] N. Karjanto, E. van Groesen, and P. Peterson, "Investigation of the maximum amplitude increase from the Benjamin-Feir Instability," Journal of the Indonesian Mathematical Society, vol. 8, pp. 39-47, 2002.

[50] R. H. M. Huijsmans, G. Klopman, N. Karjanto, and et al., "Experiments on extreme waves generation using the Soliton on Finite Background," in Proceedings Rougue Waves 2004, Brest, France, 2005.

[51] M. V. Berry and M. R. Dennis, "Phase singularities in isotropic random waves," Proceedings A, vol. 456, no. 2001, pp. 2059-2079, 2000 . 


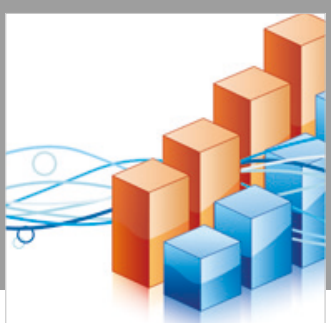

Advances in

Operations Research

\section{-n-m}
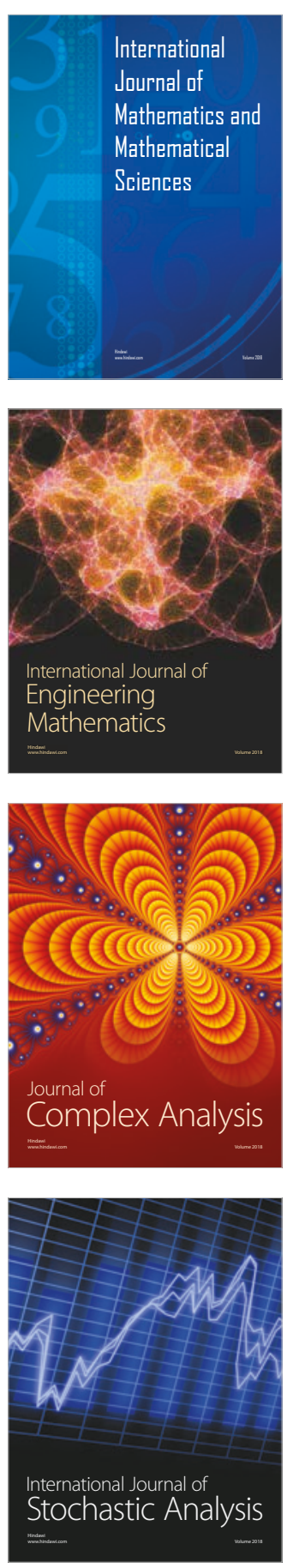
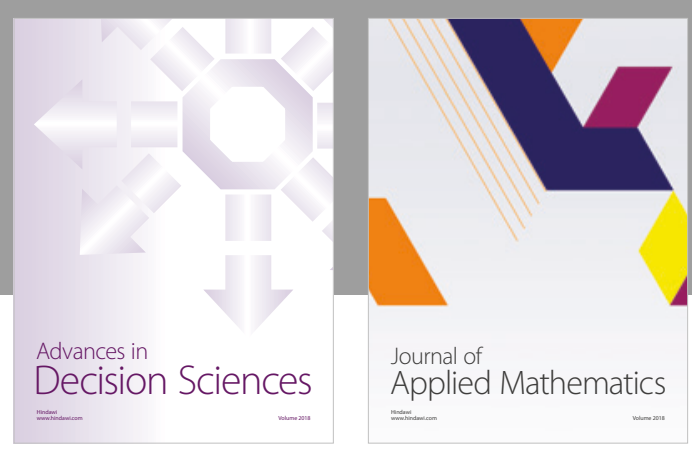

Journal of

Applied Mathematics
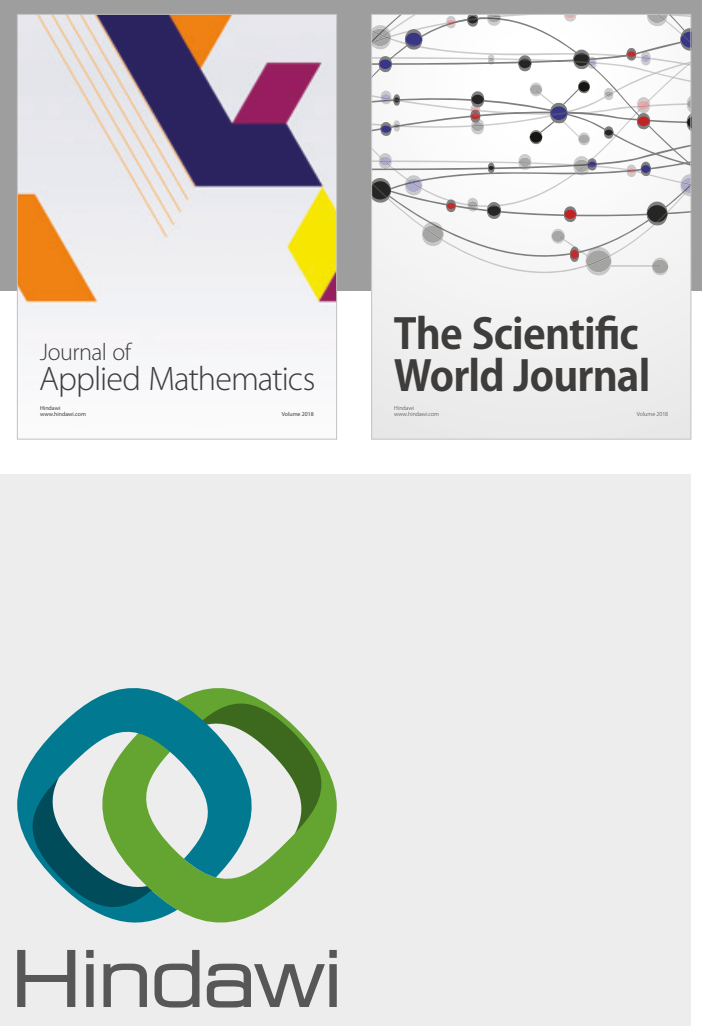

Submit your manuscripts at

www.hindawi.com

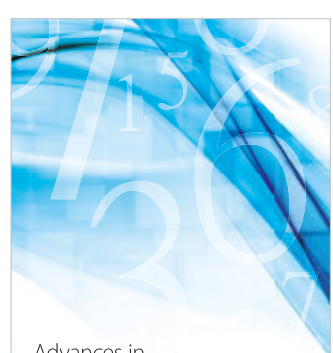

Advances in
Numerical Analysis
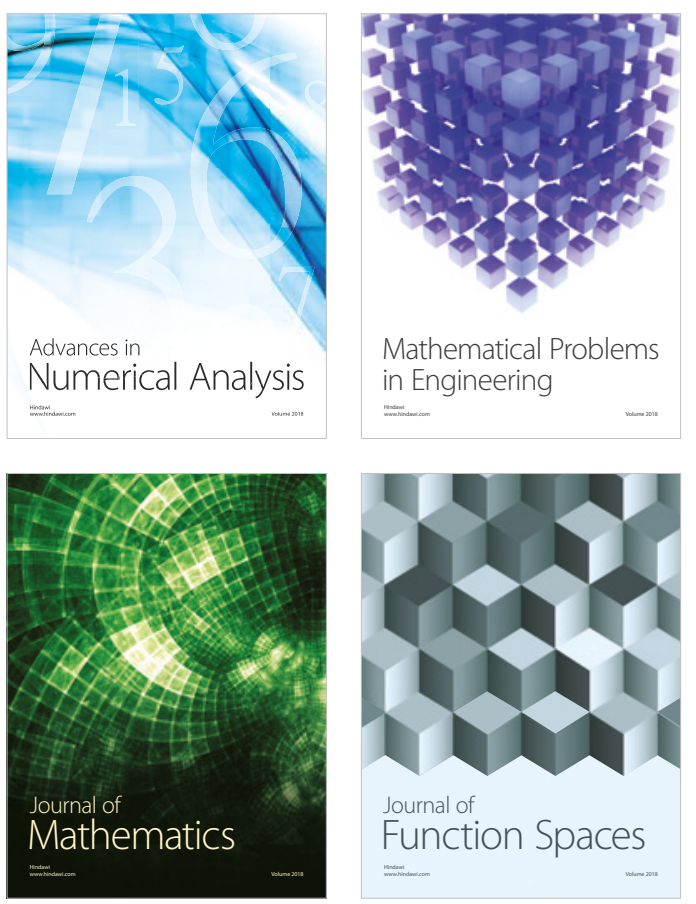

Mathematical Problems in Engineering

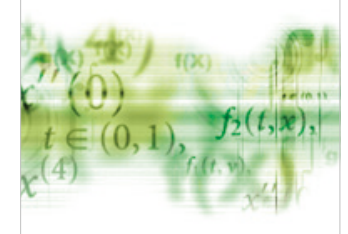

International Journal of

Differential Equations

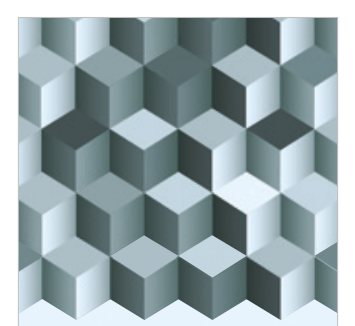

Journal of

Function Spaces

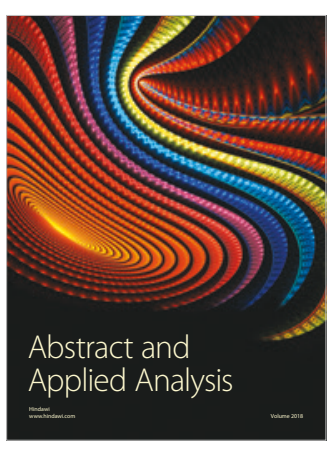

The Scientific

World Journal

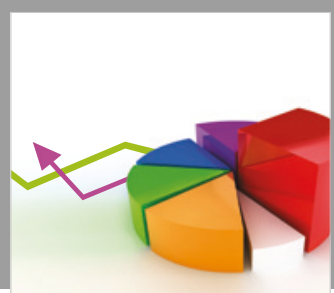

Journal of

Probability and Statistics
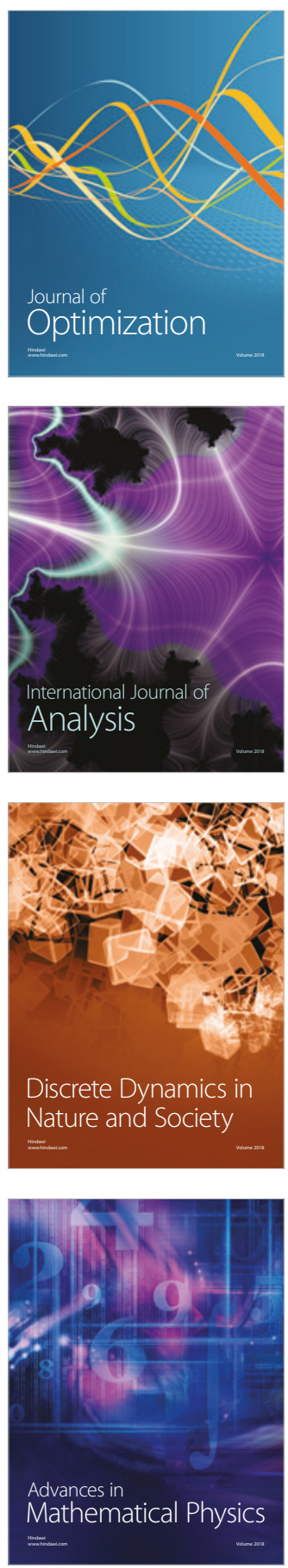